\title{
'It is just the way it was in the past before I went to test': a qualitative study to explore responses to HIV prevention counselling in rural Tanzania
}

Caoimhe Cawley ${ }^{1 *}$, Alison Wringe ${ }^{1}$, Joyce Wamoyi ${ }^{2}$, Shelley Lees ${ }^{1}$ and Mark Urassa²

\begin{abstract}
Background: Voluntary counselling and testing (VCT) for HIV first evolved in Western settings, with one aim being to promote behaviours which lower the risk of onward transmission or acquisition of HIV. However, although quantitative studies have shown that the impact of VCT on sexual behaviour change has been limited in African settings, there is a lack of qualitative research exploring perceptions of HIV prevention counselling messages, particularly among clients testing HIV-negative. We conducted a qualitative study to explore healthcare worker, community and both HIV-negative and HIV-positive clients' perceptions of HIV prevention counselling messages in rural Tanzania.
\end{abstract}

Methods: This study was carried out within the context of an ongoing community HIV cohort study in Kisesa, northwest Tanzania. Nine group sessions incorporating participatory learning and action (PLA) activities were conducted in order to gain general community perspectives of HIV testing and counselling (HTC) services. Thirty in-depth interviews (IDIs) with HIV-negative and HIV-positive service users explored individual perceptions of HIV prevention counselling messages, while five IDls were carried out with nurses or counsellors offering HTC in order to explore provider perspectives.

Results: Two key themes revolving around socio-cultural and contextual factors emerged in understanding responses to HIV prevention counselling messages. The first included constraints to client-counsellor interactions, which were impeded as a result of difficulties discussing private sexual behaviours during counselling sessions, a hierarchical relationship between healthcare providers and clients, insufficient levels of training and support for counsellors, and client concerns about confidentiality. The second theme related to imbalanced gender-power dynamics, which constrained the extent to which women felt able to control their HIV-related risk.

Conclusion: Within the broader social context of a rural African setting, HIV prevention counselling based on a Western model of individual-level agency seems unlikely to make a significant contribution to sexual behaviour change until there is greater recognition by counsellors of the ways in which power dynamics within many relationships influence behaviour change. More culturally relevant counselling strategies and messages and infrastructural improvements such as additional training for counsellors and counselling rooms which ensure privacy and confidentiality, may lead to better outcomes in terms of sexual risk reduction.

Keywords: HIV testing and counselling, HIV prevention counselling, sexual behaviour, Sexual risk reduction, HIV prevention, Tanzania

\footnotetext{
* Correspondence: caoimhe.cawley@lshtm.ac.uk

'London School of Hygiene and Tropical Medicine, Keppel Street, London

WC1E 7HT, UK

Full list of author information is available at the end of the article
} 


\section{Background}

Voluntary counselling and testing (VCT) for HIV first evolved in Western settings, with a strong focus on coping and support mechanisms for those diagnosed HIV-positive, as well as on the promotion of behaviours to lower the risk of onward transmission or acquisition of HIV [1]. While there is a paucity of evidence to guide the theoretical constructs upon which HIV prevention counselling advice is expected to act, many individuallevel models of behaviour change focus on aspects of individual agency such as intentions, attitudes, beliefs and feelings of self-efficacy regarding sexual risk reduction [2]. However, social and cultural norms, as well as structural factors such as poverty, are likely to be important determinants of behaviour in African and other settings where identity is strongly influenced by family, community and other social factors [3].

As Western HIV testing norms have been imported into African settings, this has been accompanied by assumptions that, as in Western settings, counselling advice can help to encourage reductions in high risk sexual behaviours such as multiple concurrent partnerships or increases in the frequency and consistency of condom use $[4,5]$. The sharing of personal information by the client with the counsellor is seen as essential to the counselling process, as is the joint identification of risk behaviours and possible strategies to reduce them $[1,6]$. A number of studies have highlighted some of the challenges associated with the provision of HIV risk reduction counselling in African settings, including difficulties experienced by counsellors when trying to balance the contrasting aims of VCT as a public health intervention with specific goals, but also an individually targeted counselling intervention which aims to respect client autonomy and choice [7]. Other challenges include difficulties adapting standard HIV testing guidelines to local settings $[8,9]$, and difficulties and discomfort experienced by counsellors when talking with clients about sexuality and sexual behaviour $[10,11]$. Quantitative evidence to support the role of HIV testing and counselling (HTC) in sexual behaviour change in sub-Saharan Africa is weak, particularly among HIV negative individuals [12-14]. While a few qualitative studies have explored clients' perspectives of and responses to HIV prevention counselling messages in African settings, many of these have focussed on HIV-positive individuals [15-17] and few have included individuals testing HIV-negative $[18,19]$.

In order to better understand the processes through which HTC may contribute to sexual behaviour change and HIV prevention in a rural area of northwest Tanzania, we conducted a qualitative study to explore healthcare worker, community and both HIV-negative and HIV-positive clients' perceptions of HIV prevention counselling advice provided at VCT or at an antenatal provider-initiated testing and counselling (PITC) service (antenatal PITC has a greater focus on diagnosis and referral of HIV-positive patients compared to VCT, but still includes counselling on HIV risk reduction regardless of HIV-status [20]).

\section{Methods}

\section{Study setting and design}

This study was carried out within the context of a community HIV cohort study in Kisesa in rural northwest Tanzania, where regular rounds of demographic and HIV serological surveillance (sero-surveys) have been ongoing since 1994 [21]. HIV prevalence in the study area was estimated at $6.5 \%$ in 2010, and most residents are subsistence farmers. VCT services were first provided as part of the 2003-2004 sero-survey, with further provision of VCT during three additional sero-survey rounds between 2006 and 2013. A permanent VCT clinic opened within the study area's main health centre in 2005, while PITC has been routinely offered to pregnant women attending the health centre's antenatal clinic (ANC) since the end of 2008. Temporary outreach VCT services are occasionally provided by governmental or non-governmental organisations. Although they have improved over time, rates of HIV testing uptake in Kisesa remain lower than the national average, with coverage of testing being approximately $40 \%$ among men and $50 \%$ among women in 2010 [22], compared to $47 \%$ among men and $62 \%$ among women in the country as a whole in 2011/2012 [23].

Qualitative data collection took place between February and April 2012. Nine group activities incorporating participatory learning and action (PLA) activities were conducted in order to gain general community perspectives of HTC services, as well as to serve as a source of recruitment for in-depth interviews (IDIs). Thirty IDIs with HIVnegative and HIV-positive service users explored individual experiences of completing HTC, while five IDIs were carried out with nurses or counsellors offering HTC in order to explore provider perspectives. The IDIs with service users and healthcare workers provided the richest source of information in terms of understanding responses to HIV prevention counselling messages, and so the majority of findings reported here are drawn from these.

\section{Sampling, recruitment and data collection}

PLA activities were carried out with four female and five male groups of six to twelve participants, stratified by rural or urban residence and age-group (18-34 or 35-60 years). A combination of purposive sampling and snowball techniques were used. Firstly, four to six individuals were purposively selected from a sampling frame constructed from the demographic and sero-surveillance 
datasets, which included some 'seeded' individuals who were known or reported to have used HTC services, as well as some who had not used HTC. The 'seeded' focus group approach has previously been used in this setting [24], and allowed us to include HTC clients in the group activities without inadvertently revealing their HTC-use status during recruitment or data collection activities. Next, each purposively sampled individual was asked to invite a neighbour or friend from their village from the same age group to participate. In total 48 purposively sampled men and women were invited to participate, of whom 40 attended and 32 brought a neighbour or friend, resulting in a total of 72 participants (41 men and 31 women). The PLA activities included brainstorming activities using flip-chart paper to express ideas and opinions relating to the perceived usefulness of HTC services, positive and negative aspects of service provision, and opinions relating to counsellors.

IDIs were carried out with 15 men and 15 women who had used HTC services, 10 of whom were HIVpositive (five men and five women). The majority of interviewees $(n=25)$ were recruited via the group activities. At the end of the activity, each participant was asked privately whether s/he was willing to discuss previous use of HTC. If the participant agreed and was an HTC user, s/he was invited to participate in an IDI on experiences of going for HTC. The discussion guide included topics on motivations for going for HTC, impressions of counsellors, information and advice shared with and received from counsellors, attitudes and feelings regarding the counselling advice received and regarding sexual risk reduction, and experiences of disclosing HTC use to partners or other family members. Five HIVpositive individuals were recruited for IDIs via the HIV care and treatment clinic (CTC) at Kisesa health centre in order to recruit individuals who may and may not have accessed antiretroviral therapy.

Five IDIs were carried out with healthcare workers, purposively sampled to include individuals providing $\operatorname{VCT}(n=2)$, PITC at the ANC $(n=2)$, and one member of the District Health Management Team who provided training, supervision and support for counsellors. Topic guides for healthcare workers providing HTC services included length of time and experience providing HTC, the content and information shared during pre and posttest counselling sessions, community perceptions of HTC, and levels of training and support received. The IDI with the HTC trainer covered issues relating to the content of counsellor training programmes, as well as ongoing professional development, support and supervision for counsellors.

All group activities and IDIs were conducted in Swahili by same gender researchers, with the exception of the IDI with the HTC trainer (a woman who was interviewed by a man). Study activities were audio-recorded following informed consent from participants, and then transcribed and translated into English.

\section{Data analysis}

Coding and analysis of transcripts was carried out in Nvivo10, guided by a framework approach [25]. After familiarisation with the data, an initial coding frame was developed based on the research questions, but also drawing on concepts derived from theories of behaviour change. Codes were also generated inductively, grounded in the accounts of study participants. The initial coding frame developed by the lead author (CC) was reviewed by co-authors (AW, SL and JW) and revised according to emerging concepts, which were grouped under overarching themes. The refined coding-frame was then applied to all transcripts. Data were then synthesized, distilling the coding to explore the emerging patterns and meanings in terms of sexual behaviour change and HIV-prevention, and to provide explanations for findings.

\section{Results}

The characteristics of the 30 HTC clients who completed IDIs are shown in Table 1. Most clients had received their most recent $\mathrm{HTC}$ at the walk-in VCT clinic at Kisesa Health Centre and had tested within the last year. In total 18 were HIV-negative, 10 were HIVpositive and two were of unknown HIV status.

Two key themes emerged in understanding responses to HIV prevention counselling messages in Kisesa. These revolved around socio-cultural and structural factors, including i) constraints to client-counsellor interactions, and ii) gender norms and relationship dynamics. We found only moderate support for the idea that individual attributes such as intentions or feelings of self-efficacy had an impact on behaviour change following HTC, as perceptions of HIV counselling advice were affected by the broader socio-cultural and contextual factors that shaped participants' lives. Findings are presented below in each of the two key thematic areas.

\section{Constraints to client-counsellor interactions Difficulties talking about sexual behaviour}

Difficulties in discussing sexuality were frequently reported by IDI participants, with apparent barriers in terms of clients sharing details of their private sexual lives with counsellors. This appeared to be particularly so for women, few of whom seemed to engage fully in counselling sessions by sharing detailed personal information with counsellors, or by seeking advice on specific issues. Some woman appeared unaware of the right to pro-actively seek information or advice, mentioning that counsellors should be the main speakers during the conversation. Women also appeared 
Table 1 Characteristics of HTC users participating in IDIs

\begin{tabular}{|c|c|c|}
\hline & Male $(n=15)$ & Female $(n=15)$ \\
\hline \multicolumn{3}{|l|}{ Age group } \\
\hline $18-24$ & $4(26.7 \%)$ & $3(20.0 \%)$ \\
\hline $25-34$ & $2(13.3 \%)$ & $4(26.7 \%)$ \\
\hline $35-44$ & $4(26.7 \%)$ & $4(26.7 \%)$ \\
\hline$>45$ & $5(33.3 \%)$ & $4(26.7 \%)$ \\
\hline \multicolumn{3}{|l|}{ Location of most recent HTC } \\
\hline $\begin{array}{l}\text { Community outreach-VCT } \\
\text { (during sero-survey) }\end{array}$ & $1(6.7 \%)$ & \\
\hline Walk-in VCT clinic (Kisesa health centre) & $8(53.3 \%)$ & $9(60.0 \%)$ \\
\hline Other $V C T^{a}$ & $4(26.7 \%)$ & $3(20.0 \%)$ \\
\hline Antenatal PITC & & $3(20.0 \%)$ \\
\hline Unknown & $2(13.3 \%)$ & \\
\hline \multicolumn{3}{|l|}{ Time since most recent HTC } \\
\hline$<1$ year & $9(60.0 \%)$ & $7(46.7 \%)$ \\
\hline $2-4$ years & $4(26.7 \%)$ & $3(20.0 \%)$ \\
\hline$>4$ years & & $4(26.7 \%)$ \\
\hline Unknown & $2(13.3 \%)$ & $1(6.7 \%)$ \\
\hline \multicolumn{3}{|l|}{ HIV Status } \\
\hline Negative & $9(60.0 \%)$ & $9(6.00 \%)$ \\
\hline Positive & $5(33.3 \%)$ & $5(33.3 \%)$ \\
\hline Unknown & $1(6.7 \%)$ & $1(6.7 \%)$ \\
\hline \multicolumn{3}{|l|}{ Area of residence } \\
\hline Rural & $7(46.7 \%)$ & $9(60.0 \%)$ \\
\hline Trading centre (urban) & $8(53.3 \%)$ & $6(40.0 \%)$ \\
\hline \multicolumn{3}{|l|}{ Level of education } \\
\hline None & $1(6.7 \%)$ & \\
\hline Primary & 7 (46.7 \%) & 13 (86.7 \%) \\
\hline Secondary or higher & 7 (46.7 \%) & $2(13.3 \%)$ \\
\hline \multicolumn{3}{|l|}{ Unknown } \\
\hline \multicolumn{3}{|l|}{ Marital status } \\
\hline Married monogamous & 7 (46.7 \%) & $9(60.0 \%)$ \\
\hline Married polygamous & $1(6.7 \%)$ & \\
\hline Separated/divorced & $2(13.3 \%)$ & $2(13.3 \%)$ \\
\hline Widowed & $2(13.3 \%)$ & $3(20.0 \%)$ \\
\hline Single (never married) & $3(20.0 \%)$ & $1(6.7 \%)$ \\
\hline
\end{tabular}

hesitant to ask questions or share information as a result of the sensitive nature of the subject matter.

I didn't ask her questions, she is the one who asked me if I have a husband. I didn't have any question to ask her. I wondered what kind of question I could ask, I didn't want to ask. (HIV-positive female, 45-60 years).
A number of women reported having met with a male counsellor, and this appeared to make discussion of private sexual matters particularly difficult.

Interviewer: What did you talk to him [the counsellor] about?

Respondent: It is him who was asking as I answer... Actually I never thought in my mind to ask him any question... in general it was hard to tell a man. We could talk about other things but for...mmm it is difficult. It is just fear to tell the male counsellor, because all men are... they are of the same calibre. (HIV-negative female, 18-29 years).

Compared to women, men more often reported feeling at ease during counselling sessions and more commonly mentioned that they had free and open discussions with counsellors.

I felt free to talk to him as a result of the way he also talked to me. Then at once the fear I had went away and I was able to tell him about my problem... you have to explain to him so that he can be able to have a clear picture about your issues..." (HIV-negative male, 18-29 years).

Some men appeared to feel at ease even if they met with a counsellor of the opposite sex. However, one man admitted that he withheld information from a female counsellor because he appeared to have been embarrassed or ashamed about an extra-marital affair he had had. This limited the potential contribution of counselling in terms of identifying possible strategies or plans for behavioural change.

I never wanted to be transparent because she was an expert...she tried to interrogate me why I had gone there, I told her that I just felt like coming to test to check on my health, even though I knew I had an extra marital affair one day. I never told her about that. (HIV-negative male, 30-44 years).

\section{Hierarchical relationship between clients and counsellors}

Counsellors recounted their efforts to engage clients in open discussions about their problems, and reported being dedicated to their profession. However, counsellors also reported that they felt a responsibility to help reduce the spread of HIV and to educate their clients, and were sometimes authoritative and directive in their counselling, creating a hierarchical relationship between healthcare provider and 'patient'.

You have to ensure that somebody has understood.... It is until he repeats everything that you have said.... and then if you realize that it is correct, then he would 
have understood.... You continue to advise him that it is supposed to be like this and this (Healthcare worker number 3).

Clients reported that counsellors were kind, caring and knowledgeable, but also referred to them as 'experts', and stated their intentions to comply with the 'rules' or 'instructions' which they received. Counsellors' roles as experts seemed to place them in a position of authority and respect, and this is likely to have influenced clients' perceived abilities to ask questions or share information freely during counselling sessions.

What I know about the guiding and counselling, it is somewhere we go so as to be given instructions. (Male group activity participant, age-group 35-60, rural residence).

I told her that I will receive them [the test results] despite what they will be, if it is HIV positive then you will tell me the right procedure to follow.

(HIV-negative male, 18-29 years).

Counsellors occasionally highlighted difficulties interacting with clients or engaging them in two-way discussions. One counsellor mentioned that clients were 'always open', although she later described the case of a woman who 'was not able to put it open that she had intercourse contrary to the way it was supposed to be done' (Healthcare worker number 1), implying a certain moral overtone or judgement of her client's behaviour which may have discouraged further discussion.

\section{Confidentiality}

Concerns about confidentiality may affect client willingness to share personal information during HTC. Most study participants reported that they felt counsellors were trustworthy and were unlikely to disclose test results or confidential information to others. However, a small number of participants reported concerns about counsellor confidentiality, including one HIV-positive woman who explained how a family member was informed about her HIV-positive status before she had received the result herself. During one group activity, male participants referred to rumours that some counsellors did not respect privacy and 'gossiped' about patients.

A few participants stated that a client's HIV status could sometimes be deduced by other clients in the waiting room if an individual spent a long time with the counsellor (assumed to be HIV-positive). This may make clients reluctant to engage in lengthy counselling sessions for fear others might suspect them of being HIV-positive. During PLAs, several participants raised concerns that the counselling rooms at the health centre were not sufficiently private and that conversations between counsellors and clients might be overheard by other patients or staff. This inhibited communication and was particularly a problem at the ANC, where HTC sessions were conducted in an only partially private area.

If the nurse and the patient talk loudly, the other people might hear. But if we had a room that was covered like this one, you could talk to the mother inside here very well. (Healthcare worker number 2).

\section{Training and support for counsellors}

Healthcare workers were generally appreciative of the training they had received. However they reported that training courses were too short, and that they had not received any refresher training sessions or supervision, but that these would be useful in order to help them update and renew their skills. One nurse working at the ANC reported that she had started providing PITC before receiving any official training and that this had made her work difficult, particularly because she was unsure about how to deliver HIV-positive test results to clients.

That time was not enough... There were many things to be learnt in two weeks... So they should have made it to be one month. It will have been better because we could learn slowly. And put all the things straight. Or the refresher courses should be there, so that people can be reminded. (Healthcare worker number 3).

Many clients reported that they had received standardised 'ABC' messages from counsellors (abstain from sex, be faithful to one partner and/or use condoms), but there was generally little evidence of individual tailoring of counselling messages to clients' particular circumstances. There also appeared to be limitations to the depth of the discussions held during counselling sessions. This is highlighted by the case of one young woman who, in response to a recommendation to use condoms, reported that she felt her partner would not be willing to do so. The counselling recommendation was to deny her partner sex, but did not seem to include a broader discussion of potential strategies to attempt to engage her partner in a dialogue on the topic.

He told me to use the condom... I asked him what if he refuses, if he refuses to use the condom. "Don't have sexual intercourse with him so that he can come and test". (HIV-negative woman 18-29 years).

\section{Gender norms and relationship dynamics}

Men commonly reported being satisfied with the counselling advice they had received, and seldom seemed to anticipate difficulties adhering to it. In contrast, women 
often highlighted concerns about revealing their attendance at HTC to their partners or about trying to discuss the advice received, reporting fears that their partners would not be willing to engage in the conversation or that they would be unhappy about certain aspects of the advice (for example recommendations to use condoms). Men and women's perceptions of the counselling advice they received highlighted inequitable gender roles, with men feeling empowered after having gone for HTC, but women often feeling anxious or indifferent about their ability to act upon counselling advice.

\section{I actually felt happy because he advised me on important issues, they got in to my mind, and I accepted it that actually this advice was important to me to use it. I really felt happy. (HIV-negative male, 18-29 years).}

You might get home and explain to your partner. When he doesn't listen it becomes difficult. Even if I start to advise him by telling him that, please partner it is like this and this, he doesn't want to understand. (HIV-negative woman, 30-44 years).

\section{He [the counsellor] asked if I have ever used the} condom, then I replied that I hadn't... There was not enough elaboration because some of us can't use protection. .... Some men don't want to use the condoms. (HIV-negative female, 18-29 years).

Suspicions of partner infidelity were common, and many participants reported feeling at risk of HIV as a result of their partner's rather than their own behaviour. In particular, women reported that it was expected or accepted that men could have multiple partners, and seemed to imply there was a certain inevitability to their partners' infidelity, but that there was little they could do to change this.

You should get home and start telling your partner. He listens to you during that time but after some time he forgets about it. He starts doing the same thing [having extra-marital partners]. (HIV-negative woman 30-44 years).

Several women implied that it was the testing aspect of the service, rather than the counselling offered, which was most useful. One young woman described that not much had changed in her relationship after going for HTC, despite the fact that she suspected her husband of being unfaithful, but that she planned to test again the next month because she found the testing process reassuring as a way to check up on her HIV status.
It is just the way it was in the past before I went to test... I just encouraged myself for the next month to come to go and test to be able to check my health status so as to continue protecting myself. (HIVnegative woman 18-29 years).

Some participants reported having gone for HTC together with their partner, either because they were planning to get married or because they were pregnant, while a small number reported having gone to test together simply to confirm each other's HIV status. A number of participants reported that couples testing would be useful to do, either because it might facilitate better commitment to sexual risk reduction by both members of the couple, and/or because it would help couples to learn about each other's HIV status.

After receiving all that guiding and counselling I just felt good, but there is one thing that I felt was not good, it is that I didn't come along with my wife. I felt that it would have been better if we would have been together with my wife. (HIV-negative male, 45-60 years).

Maybe that is where we get enlightened and be able to say that, we are both mobilised. It is not that I get motivated alone. (HIV-negative female 30-44 years).

\section{Discussion}

Our study highlighted several barriers, influenced by social and contextual factors, which seemed to limit plans or intentions regarding sexual behaviour change following HTC, including limited communication between clients and counsellors during counselling sessions, and inequitable gender-power relations.

Previous studies in sub-Saharan Africa have highlighted counsellors' difficulties talking about sexuality or intimate matters during HTC, for example because the topic was embarrassing or difficult to talk about $[10,11]$, and it is likely that clients share similar concerns. In some African cultures there are prescribed social norms about who should be consulted for advice regarding sexual matters, and involving an outsider in family problems may go against traditional practice [26]. Such findings may help to explain why candid discussions between clients and counsellors appeared difficult for many participants in our study, and indicate that counselling approaches which include techniques to put clients and counsellors at ease when talking about sexuality would be useful.

Previous qualitative research in Tanzania and elsewhere in sub-Saharan Africa has noted the dominant role of men within relationships [18, 27], and that men's sexual respectability is often linked to greater sexual activity and the conquest of multiple partners, in contrast to women's sexual respectability which is characterised 
by obedience, faithfulness and subservience to male partners $[28,29]$. One study in Tanzania reported that men may exaggerate their numbers of sexual partners, while women were more self-effacing and tended to underreport non-marital partnerships [30]. These findings may help to explain why men in our study appeared more at ease discussing their sexual behaviour during HTC compared to women, and why men felt more empowered to act upon counselling advice.

A hierarchical relationship between healthcare provider and 'patient' was seen to inhibit communication between both male and female clients and counsellors during HTC, and other studies in sub-Saharan Africa have noted similar hierarchical relationships $[9,31,32]$. Lie et al. [33] note that HIV counselling guidelines tend to be prescriptive and directive by endorsing preferred behaviours (e.g. using condoms, remaining faithful to one partner), in contrast to more traditional personcentred counselling approaches which have a greater focus on client autonomy. As also observed in this study, counsellors seem to face challenges when trying to balance the contrasting aims of HTC as both a public health intervention with specific goals (i.e. to encourage sexual risk reduction), but one that is simultaneously meant to respect individual clients' choices [7]. Training courses and HIV counselling guidelines which place greater emphasis on maintaining a neutral stance, and on techniques for engendering more equitable clientcounsellor interactions, may help to improve the outcome of HTC in terms of sexual risk reduction. For example, one study in South Africa noted that counsellors who attempted to engage clients by 'making appeals' rather than 'prescribing rules for living' seemed to be more successful in gaining clients' engagement and commitment to sexual risk reduction [7].

Research has suggested that in sub-Saharan Africa, family and community are more central to the construction of health and well-being than the individual $[3,9]$. In support of this, one study reported that HIV-positive Ugandan men and women's main motivations in preventing HIV transmission were altruistic wishes to protect sexual partners and to avoid orphaning children [34]. In African settings, HIV training modules and counselling messages which focus on broader culturally relevant altruistic messages relating to partners, children and the wider community, for example emphasising the benefits of protecting unborn children from HIV, or of maintaining the integrity and health of the family unit, may be more successful than ones which focus solely on individual interest [34].

Although studies have reported that counsellors sometimes struggle to balance confidentiality requirements with what they may perceive as being in the best interest of the patient or the community at large (for example, by disclosing an HIV-positive individual's status to a partner or other family members without the patient's consent, in order that the partner may protect himself or herself from HIV, or that the family may provide care and support for the patient) $[8,35]$, other studies have shown that confidentiality is of paramount importance for clients [9, 33]. Although most participants in our study reported that they felt counsellors were trustworthy, even small fears relating to breaches of confidentiality may have a disproportionate impact upon clients' willingness to share sensitive information. Thus counsellor training modules should cover the importance of maintaining client confidentiality in sufficient detail, while clinic buildings and infrastructure should be sufficiently private so as to put clients (and counsellors) at ease.

As highlighted by counsellors in this study, additional aspects of counsellor training which require attention include the depth in which topics are covered, and the availability of refresher training courses and supervision and support for counsellors. Furthermore, it was also apparent that the risk reduction strategies proposed by counsellors were often limited to only include condom use or abstention, and tailored discussions that covered a fuller range of available, relevant HIV prevention tools (e.g. HIV testing for partners, treatment for partners who test positive, testing and treatment for sexually transmitted infections, and eventually, pre-exposure prophylaxis) which might represent more realistic prevention methods for some women, should also be covered in counsellors' training courses.

A number of clients reported finding the process of learning their HIV status reassuring in itself, regardless of counselling messages received. However, this knowledge alone seemed unlikely to lead to reductions in sexual risk behaviour. Several participants reported the desire to know their HIV status as their main motivation for going for HTC, and although counselling sessions were not observed as part of this study, it is possible that a strong focus of the counselling advice was on the different possible HIV test results and how they should be interpreted, rather than on sexual risk reduction. Previous studies have suggested that 'de-linking' testing and counselling service provision might help clients to focus on counselling messages and thus more effectively support behaviour change $[19,36]$, but this hypothesis remains untested.

A relatively small number of clients reported having gone for testing together with their partner, however some expressed a desire to do so. The few individuals who reported having gone for couples-HTC reported positive experiences, including that couples might get 'motivated' together. Although previous studies have shown that couples-counselling is challenging for counsellors, particularly in the case of sero-discordant results [37, 38], others have reported the approach to be 
beneficial because discussions between husband and wife can be mediated by a third party, and because the process can help to renew commitment to the relationship by both members of the couple [39]. Much of the focus of VCT in sub-Saharan Africa to date has been on the provision of an individual service, however couplescounselling likely represents a promising option in terms of increasing the uptake of HTC services as well as helping to encourage behavioural change [40].

A limitation of this study was that HTC users may have been reluctant to share details of what was discussed with counsellors with study researchers, due to the sensitive nature of the subject matter, and due a similar hierarchical relationship between participants and researchers to that observed between clients and counsellors. In order to minimise this bias, interviewing techniques to build rapport and trust with participants were addressed during fieldworker training. In order to maximise confidentiality, all IDIs were conducted either in private rooms near Kisesa Health Centre, or in the case of rural residents, in private locations near rural dispensaries. For confidentiality reasons, individual counselling sessions were not observed as part of this study. Had we been able to do so, we might have gained more a more in-depth understanding of the nature of the interactions between clients and counsellors during counselling sessions, and of the topics covered.

\section{Conclusion}

Within the broader socio-cultural context of a rural African setting such as Kisesa, HIV prevention counselling alone based on a Western model of individual-level agency is unlikely to make a significant contribution to sexual behaviour change. More culturally relevant counselling strategies and messages, such as those which aim to put clients and counsellors at ease when discussing sexuality or which emphasise altruistic wishes to reduce the spread of HIV, and infrastructural improvements such as additional training for counsellors and counselling rooms which ensure privacy and confidentiality, may lead to better outcomes in terms of sexual risk reduction. In the long-term, structural interventions such as improved access to education might help to reduce gender-imbalanced power dynamics and to improve outcomes among women.

\footnotetext{
Abbreviations

ANC: antenatal clinic; CTC: care and treatment clinic; HTC: HIV testing and counselling; IDI: in-depth interview; PITC: provider-initiated testing and counselling; PLA: participatory learning and action activities; VCT: voluntary counselling and testing.
}

\section{Acknowledgements}

We would like to thank the study participants, as well as the clinicians and healthcare workers at Kisesa Health Centre, without whom this research would not have been possible. Thanks are also due to the research assistants involved in data collection (Ray Nsigaye, Joyce Chuwa, Grace Bulugu,
Mpyanjo (hagu) and the wider staff at the National Institute of Medical Research in Mwanza who are involved in running the Kisesa cohort study.

\section{Funding}

CC is funded by the UK Economic and Social Research Council. AW is funded by a Population Health Scientist award, jointly funded by the UK Medical Research Council (MRC) and the UK Department for International Development (DFID) under the MRC/DFID Concordat agreement and is also part of the EDCTP2 program supported by the European Council. Data collection activities for this study were funded by the Gordon Smith Travelling Scholarship from the London School of Hygiene \& Tropical Medicine. The Kisesa observational HIV cohort has been funded by the Netherlands Government through the TANESA Project and Global Fund Round 4 (Grant: TNZ-405-GO4-H) and Round 9 (Grant: TNZ-911-G14-S), through the Tanzania National Coordinating Mechanism (TNCM). No funding bodies were involved in the design, collection, analysis or interpretation of data for this study, nor in the decision to submit for publication.

\section{Availability of data and materials}

Data may be available upon request by contacting

alison.wringe@lshtm.ac.uk, subject to review by the ethics committees of the Tanzanian Medical Research Coordinating Committee and the London School of Hygiene and Tropical Medicine.

\section{Authors' contributions}

CC and AW conceived of the study. CC designed the study in collaboration with AW, JW and MU, including designing the sampling and recruitment methods and developing the data collection tools. CC oversaw data collection, analysed the data and wrote the manuscript with inputs from AW, JW and SL. All authors read and approved the final manuscript.

\section{Competing interests}

The authors declare that they have no competing interests.

\section{Consent for publication}

Consent for the use of anonymous quotations in publications was obtained from all IDI and group activity participants.

\section{Ethics approval and consent to participate}

This study was approved by the ethics committees of the Tanzanian Medical Research Coordinating Committee (application number NIMR/HQ/R.8a/Nol.IX/130) and the London School of Hygiene and Tropical Medicine (application number 6036). During group activities verbal consent (for participation, audio-recording of study activities and use of anonymous quotations in publications) was obtained for IDls the same consent was obtained in either verbal or written format depending on participant preference. Fictitious names were used during group activities to safeguard anonymity, and participants were advised that they were not expected to share personal information, particularly with regard to HIV-status or prior HTC use. Recruitment procedures for IDIs using the seeded focus group approach were designed to maximise confidentiality and avoid any inadvertent disclosure of HTC use status. Some 'seeded' HTC users were HIV-positive, however field workers were unaware of the HIV status of participants unless they chose to disclose this during IDIs. For HTCusers recruited via the $C T C$, prior to agreeing to participate patients were informed that interviewers would be aware of their HIV-status, due to the nature of their recruitment

\section{Author details}

'London School of Hygiene and Tropical Medicine, Keppel Street, London WC1E 7HT, UK. ${ }^{2}$ National Institute for Medical Research, Mwanza, Tanzania.

Received: 16 October 2015 Accepted: 14 May 2016

Published online: 08 June 2016

\section{References}

1. Centers for Disease Control (CDC). Additional recommendations to reduce sexual and drug abuse-related transmission of human T-lymphotropic virus type III/lymphadenopathy-associated virus. MMWR. 1986;35(10):152-5.

2. Denison JA. Behaviour change: A summary of four major theories. Arlington: Family Health International; 1996. 
3. Airhihenbuwa CO, Obregon R. A critical assessment of theories/models used in health communication for HIV/AIDS. J Health Commun. 2000;5:5-15.

4. WHO. Source book for HIV/AIDS counselling training. Geneva: WHO; 1994.

5. Ministry of Health. National Guidelines for Voluntary Counselling and Testing, 2005. Tanzania: Tanzanian Ministry of Health and Social Welfare; 2005.

6. UNAIDS. Counselling and HIV/AIDS. Geneva: UNAIDS Technical Update; 1997.

7. van Rooyen $\mathrm{H}$, Durrheim K, Lindegger $\mathrm{G}$. Advice-giving difficulties in voluntary counselling and testing: a distinctly moral activity. AIDS Care. 2011;23(3):281-6.

8. Angotti N. Working outside of the box: how HIV counselors in Sub-Saharan Africa adapt Western HIV testing norms. Soc Sci Med. 2010;71(5):986-93.

9. Angotti N. Testing differences: the implementation of Western HIV testing norms in sub-Saharan Africa. Cult Health Sex. 2012;14(4):365-78.

10. Baggaley R, Sulwe J, Kelly M, MacMillan MN, Godfrey-Faussett P. HIV counsellors' knowledge, attitudes and vulnerabilities to HIV in Lusaka, Zambia, 1994. AIDS Care. 1996;8(2):155-66.

11. Buwalda P, Kruijthoff DJ, de Bruyn M, Hogewoning A. Evaluation of a homecare/counselling AIDS programme in Kgatleng district, Botswana. AIDS Care. 1994;6(2):153-60.

12. Denison JA, O'Reilly KR, Schmid GP, Kennedy CE, Sweat MD. HIV voluntary counseling and testing and behavioral risk reduction in developing countries: a meta-analysis, 1990-2005. Aids Behav. 2008;12(3):363-73.

13. Fonner VA, Denison J, Kennedy CE, O'Reilly K, Sweat M. Voluntary counseling and testing (VCT) for changing HIV-related risk behavior in developing countries. Cochrane Database Syst Rev. 2012;9:Cd001224.

14. Kennedy CE, Fonner VA, Sweat MD, Okero FA, Baggaley R, O'Reilly KR. Provider-Initiated HIV Testing and Counseling in Low- and Middle-Income Countries: A Systematic Review. Aids Behav. 2012

15. Allen C, Mbonye M, Seeley J, Birungi J, Wolff B, Coutinho A, et al. ABC for people with HIV: responses to sexual behaviour recommendations among people receiving antiretroviral therapy in Jinja, Uganda. Cult Health Sex. 2011;13(5):529-43.

16. McArthur M, Birdthistle I, Seeley J, Mpendo J, Asiki G. How HIV diagnosis and disclosure affect sexual behavior and relationships in Ugandan fishing communities. Qual Health Res. 2013;23(8):1125-37.

17. Lifshay J, Nakayiwa S, King R, Reznick OG, Katuntu D, Batamwita R, et al. Partners at risk: motivations, strategies, and challenges to HIV transmission risk reduction among HIV-infected men and women in Uganda. AIDS Care. 2009;21(6):715-24.

18. Sangiwa G, Van Der Straten A, Grinstead OA, VCT Study Group. Clients' Perspective of the Role of Voluntary Counseling and Testing in HIV/AIDS Prevention and Care in Dar Es Salaam, Tanzania: The Voluntary Counseling and Testing Efficacy Study. Aids Behav. 2000;4(1):35-48.

19. Sikasote J, Grant L, Chinn DJ, Macwang'i M, Murray SA. Voluntary counselling and testing for HIV in a Zambian mining community: serial interviews with people testing negative. Sex Transm Infect. 2011;87(5):433-8.

20. Ministry of Health and Social Welfare. Guidelines for HIV testing and counselling in clinical settings. Tanzania: Tanzanian Ministry of Health and Social Welfare; 2007.

21. Boerma JT, Urassa M, Senkoro K, Klokke A, Ngweshemi JZ. Spread of HIV infection in a rural area of Tanzania. Aids. 1999;13(10):1233-40.

22. Cawley $C$, Wringe $A$, Isingo $R$, Mtenga $B$, Clark $B$, Marston $M$, et al. Low rates of repeat HIV testing despite increased availability of antiretroviral therapy in rural Tanzania: findings from 2003-2010. PLoS One. 2013;8(4):e62212.

23. Tanzania Commission for AIDS (TACAIDS) ZAC, National Bureau of Statistics, Office of the Chief Government Statistician, ICF International. Tanzania HIV/ AIDS and Malaria Indicator Survey 2011-12. Dar es Salaam, Tanzania: 2013

24. Busza J, Zaba B, Urassa M. The "seeded" focus group: a strategy to recruit $\mathrm{HIV}+$ community members into treatment research. Sex Transm Infect. 2009;85(3):212-5.

25. Pope C, Ziebland S, Mays N. Qualitative research in health care. Analysing qualitative data. BMJ. 2000;320(7227):114-6.

26. Seeley J, Wagner U, Mulemwa J, Kengeya-Kayondo J, Mulder D. The development of a community-based HIV/AIDS counselling service in a rural area in Uganda. AIDS Care. 1991;3(2):207-17.

27. Siu GE, Wight D, Seeley JA. Masculinity, social context and HIV testing: an ethnographic study of men in Busia district, rural eastern Uganda. BMC Public Health. 2014;14:33.

28. Wight D, Plummer ML, Mshana G, Wamoyi J, Shigongo ZS, Ross DA. Contradictory sexual norms and expectations for young people in rural Northern Tanzania. Soc Sci Med. 2006;62(4):987-97.
29. Krumeich A, Weijts W, Reddy P, Meijer-Weitz A. The benefits of anthropological approaches for health promotion research and practice. Health Educ Res. 2001;16(2):121-30

30. Nnko S, Boerma JT, Urassa M, Mwaluko G, Zaba B. Secretive females or swaggering males? An assessment of the quality of sexual partnership reporting in rural Tanzania. Soc Sci Med. 2004;59(2):299-310.

31. Gourlay A, Wringe A, Birdthistle I, Mshana G, Michael D, Urassa M. "It is like that, we didn't understand each other": exploring the influence of patientprovider interactions on prevention of mother-to-child transmission of HIV service use in rural Tanzania. PLoS One. 2014;9(9):e106325.

32. Rasmussen LM. Counselling clients to follow 'the rules' of safe sex and ARV treatment. Cult Health Sex. 2013;15 Suppl 4:S537-52.

33. Lie GT, Biswalo PM. Perceptions of the appropriate HIV/AIDS counsellor in Arusha and Kilimanjaro regions of Tanzania: implications for hospital counselling. AIDS Care. 1994;6(2):139-51.

34. King R, Lifshay J, Nakayiwa S, Katuntu D, Lindkvist P, Bunnell R. The virus stops with me: HIV-infected Ugandans' motivations in preventing HIV transmission. Soc Sci Med. 2009;68(4):749-57.

35. Evans C, Ndirangu E. Implementing routine provider-initiated HIV testing in public health care facilities in Kenya: a qualitative descriptive study of nurses' experiences. AIDS Care. 2011;23(10):1291-7.

36. Kipp W, Kabagambe G, Konde-Lule J. HIV counselling and testing in rural Uganda: communities' attitudes and perceptions towards an HIV counselling and testing programme. AIDS Care. 2002;14(5):699-706.

37. Grinstead OA, van der Straten A. Counsellors' perspectives on the experience of providing HIV counselling in Kenya and Tanzania: the Voluntary HIV-1 Counselling and Testing Efficacy Study. AIDS Care. 2000;12(5):625-42.

38. Mavhandu-Mudzusi AH, Netshandama VO, Davhana-Maselesele M. Nurses' experiences of delivering voluntary counseling and testing services for people with HIV/AIDS in the Vhembe District, Limpopo Province, South Africa. Nurs Health Sci. 2007:9(4):254-62.

39. Musheke M, Bond V, Merten S. Couple experiences of provider-initiated couple HIV testing in an antenatal clinic in Lusaka, Zambia: lessons for policy and practice. BMC Health Serv Res. 2013;13:97.

40. Grabbe KL, Bunnell R. Reframing HIV prevention in sub-Saharan Africa using couple-centered approaches. JAMA. 2010;304(3):346-7.

\section{Submit your next manuscript to BioMed Central and we will help you at every step:}

- We accept pre-submission inquiries

- Our selector tool helps you to find the most relevant journal

- We provide round the clock customer support

- Convenient online submission

- Thorough peer review

- Inclusion in PubMed and all major indexing services

- Maximum visibility for your research

Submit your manuscript at www.biomedcentral.com/submit
(O) BioMed Central 